\title{
Explicit computation of shear three-point correlation functions: the one-halo model case
}

\author{
F. Bernardeau
}

Service de Physique Théorique, CEA/DSM/SPhT, Unité de recherche associée au CNRS, CEA/Saclay, 91191 Gif-sur-Yvette Cedex, France e-mail: fbernard@spht.saclay.cea.fr

Received 16 May 2005 / Accepted 7 June 2005

\section{ABSTRACT}

We present a method for calculating explicit expressions of the shear three-point function for various cosmological models. The method is applied here to the one-halo model in the case of power law density profiles for which results are detailed. The three-point functions are found to reproduce to a large extent patterns in the shear correlations obtained in numerical simulations and may serve as a guideline to implement optimized methods for detecting the shear three-point function. In principle, the general method presented here can also be applied for other models of matter correlation.

Key words. cosmology: theory - cosmology: dark matter - cosmology: large-scale structure of Universe

\section{Introduction}

Cosmic shear surveys offer a unique and unbiased view on the way matter clusters at cosmological scales and can then serve as a means to explore the details of the gravitational instabilities. The statistical properties of the shear field, in particular their high order correlation functions, are expected to reflect those of the matter density field (see Mellier 1999). The measurement of correlation functions in the cosmic shear surveys has been recognized as a means to break the parameter degeneracy between the density parameter of the Universe and $\sigma_{8}$ that measurements of the shear power spectra provide (Bernardeau et al. 1997; Jain \& Seljak 1997), or a way to test the gravity law at cosmological scales (Bernardeau 2004).

Matter correlation functions have been explored extensively in different regimes. A reasonable understanding of their behavior has been obtained in the quasilinear regime from perturbation theory techniques and into the nonlinear regime from phenomenological approaches (see review of Bernardeau et al. 2002a). Recently a semianalytic technique based on the halo model (Seljak 2000; Ma \& Fry 2000; Scoccimarro et al. 2001; see Cooray \& Sheth 2002 , for a recent review) has attracted a lot of attention because it can provide accurate predictions for the galaxy or matter correlation properties. So far however most of these theoretical approaches have dealt with the matter density field or with the convergence field. The latter is closely related to the density field, being a simple projection of it, but the actual observational situation is more complex. What is directly measured is the shear field (through galaxy shape measurements, see Mellier 1999, for a detailed review on the observational techniques) and it is difficult to build reliable projected density maps. This forces us to explore more deeply the high-order shear correlation patterns. These, because of their geometrical nature, are more intricate than those of the convergence maps. In particular, complex relations are expected between the correlation components because of their pseudo-vector nature and because one expects vanishing " $\mathrm{B}$ " modes in the shear field. As a result shear correlation coefficients depend in a complicated way on the basis on which they are computed and have frequent sign changes, etc. (see Schneider \& Lombardi 2003, for an exhaustive exploration of these properties). It makes the construction of methods to measure the shear three-point function difficult. However, attempts have been made to detect the shear or aperture mass high order correlation functions (Bernardeau et al. 2002b; Pen et al. 2003; Jarvis et al. 2004). Although these methods can give robust results, they may be far from optimal and therefore not completely satisfactory. One aim of this paper is to provide better foundations for the design of new methods to measure shear three-point functions.

In this paper we will the focus on the one-halo model case for the description of the matter correlation properties. The idea is not to build an accurate model for the mass distribution but to build a model good enough to reproduce the generic geometrical properties of the shear correlation patterns. The one-halo model, with a power law density profile, is a good candidate for such a task, being simple enough to allow complete computations of the correlation functions. The paper is divided as follows. The second section is devoted to a presentation of the 
mathematical tools that are applied to the one-halo model in the third section. The fourth section gives some insights into the results obtained.

\section{A complex variable representation}

What we are interested in are the statistical properties of the either the projected density field $\phi(\boldsymbol{r})$, the convergence field $\kappa(\boldsymbol{r})$ and the 2-component shear field $\left(\gamma_{1}, \gamma_{2}\right)$. In the following the plane wave approximation is used, as usual, so that the convergence and shear field are all obtained through derivative operators applied to the projected potential,

$\kappa(\boldsymbol{r})=\left(\partial_{a}^{2}+\partial_{b}^{2}\right) \phi(\boldsymbol{r})$

$\left(\gamma_{1}, \gamma_{2}\right)=\left(\partial_{a}^{2}-\partial_{b}^{2}, 2 \partial_{a} \partial_{b}\right) \phi(r)$

where $\boldsymbol{r}$ is the angular position of the point on the sky and $a$ and $b$ are the point coordinates.

In principle the convergence or shear correlations are functions of the point coordinates. Given the statistical isotropy and homogeneity of the fields those correlation functions should depend only on the relative positions and are globally rotationally invariant. Those correlation functions can therefore be written for instance as a function of the three distances or equivalently as a function of 2 distances and one angle, etc. The coordinate system advocated in this paper is different. Denote the point coordinates as $\boldsymbol{r}_{1}, \boldsymbol{r}_{2}$ and $\boldsymbol{r}_{3}$ respectively; $l_{1}, l_{2}$ and $l_{3}$ are the three lengths of the triangle they form such that $l_{3}^{2}=\left|\boldsymbol{r}_{2}-\boldsymbol{r}_{1}\right|^{2}$, etc. Then define the two ratios,

$x_{3}=\frac{l_{1}^{2}}{l_{3}^{2}} ; y_{3}=\frac{l_{2}^{2}}{l_{3}^{2}}$

and the complex variable

$z_{3}=\frac{1}{2}\left(1-x_{3}+y_{3}-\sqrt{\left(1-x_{3}+y_{3}\right)^{2}-4 y_{3}}\right)$.

$\left(1-x_{3}+y_{3}\right)^{2}-4 y_{3} \leq 0$ so that $z_{3}$ generically has a non-vanishing imaginary part. The idea is then to write all correlation functions as a function of $\left(l_{i}, z_{i}\right), i$ being either 1,2 or $3 . z_{i}$ are the components of $\boldsymbol{r}_{i}$ on the basis defined by the other two points

$z_{3} \rightarrow\left(a_{3}-\mathrm{i} b_{3}\right) / l_{3}$

As a result the derivatives with respect to point coordinates can simply be re-expressed as derivatives with respect to $z_{i}$ and $\bar{z}_{i}$ :

$\partial_{\boldsymbol{r}_{i}}=\frac{1}{l_{i}}\left(\partial_{z_{i}}+\partial_{\bar{z}_{i}},-\mathrm{i} \partial_{z_{i}}+\mathrm{i} \partial_{\bar{z}_{i}}\right)$.

This implies that the Laplacian operator, or operators that give access to the shear coordinates out of the potential field, respectively read

$$
\begin{aligned}
& \partial_{a}^{2}+\partial_{b}^{2}=\frac{4}{l^{2}} \partial_{z} \partial_{\bar{z}} \\
& \partial_{a}^{2}-\partial_{b}^{2}=\frac{2}{l^{2}}\left(\partial_{z}^{2}+\partial_{\bar{z}}^{2}\right) \\
& 2 \partial_{a} \partial_{b}=-\frac{2 \mathrm{i}}{l^{2}}\left(\partial_{z}^{2}-\partial_{\bar{z}}^{2}\right) .
\end{aligned}
$$

These equations suggest that the shear field is better written as a complex number $\gamma=\gamma_{1}+\mathrm{i} \gamma_{2}$ and

$$
\begin{aligned}
& \kappa\left(l_{i}, z_{i}\right)=\frac{1}{l^{2}} \partial_{z_{i}} \partial_{\bar{z}_{i}} \phi\left(l_{i}, z_{i}\right) \\
& \gamma\left(l_{i}, z_{i}\right)=\frac{1}{l^{2}} \partial_{z_{i}^{2}} \phi\left(l_{i}, z_{i}\right) \text { and } \bar{\gamma}\left(l_{i}, z_{i}\right)=\frac{1}{l^{2}} \partial_{\bar{z}_{i}^{2}} \phi\left(l_{i}, z_{i}\right) .
\end{aligned}
$$

Full computations with such variables will however been tractable only if change coordinates can be written in a natural way. Indeed, the transform rules that give the $z_{1}$ and $z_{2}$, complex coordinates of $\boldsymbol{r}_{1}$ and $\boldsymbol{r}_{2}$, as a function of $z_{3}$ are actually simple to compute and read,

$z_{1}=\frac{1}{1-z_{3}}, z_{2}=\frac{1}{1-z_{1}}=1-\frac{1}{z_{3}}$.

The coordinate change rules have to be completed with the following relations,

$l_{1}^{2}=l_{3}^{2} z_{1} \bar{z}_{1}, \quad l_{2}^{2}=l_{1}^{2} z_{2} \bar{z}_{2}$

that give the transformation rules for the base lengths. These rules suffice for the computations of correlation of scalar quantities, e.g. correlations between the projected potential and the convergence fields.

For the computation of correlations between shear components however, further transform rules are required. These are the ones that rotate the shear coordinates from one basis to another. After vector analysis, these changes of coordinates amount to multiply the complex shear coordinates by $\left(1-z_{3}\right) /\left(1-\bar{z}_{3}\right)$ in order to change the base $\left(\boldsymbol{r}_{1}, \boldsymbol{r}_{2}\right)$ to $\left(\boldsymbol{r}_{2}, \boldsymbol{r}_{3}\right)$ or by similar operations for the other changes (applying circular permutations to the indices).

If the projected potential correlation function is known, then the computation of either the convergence field or the shear field is straightforward. It takes only a series of proper derivatives and rotation operators applied to the potential correlation function.

This approach is particularly attractive when the latter correlation function can be written as a sum of terms of the form $f(z) f(\bar{z})$. In this case the operators act independently on each factor. As we will see next this is precisely the situation that is encountered in the one-halo model with power law profiles.

\section{Correlation functions in the one-halo model}

The halo model provides a simplified description of the matter correlation properties. It has been advocated to be an accurate approach for describing the galaxy and matter correlation properties (Sheth \& Jain 1997) and studies of the dark matter density field through halo contributions have recently been developed in Seljak (2000), Ma \& Fry (2000), Scoccimarro et al. (2001), but also applied to three-point lensing statistics (Cooray et al. 2000b; and Takada \& Jain 2003b).

In this section we will illustrate the methods sketched in the previous section for shear three-point functions using the halo model, restricting for simplicity to the one-halo term. The latter is indeed expected to dominate on small scales (see for instance Fosalba et al. 2005) where the three-point function of the shear is easiest to measure in observations. In itself the 
use of the halo model for the computation of the lensing high order correlations is not new. It has been done by Cooray \& $\mathrm{Hu}$ (2001) for the convergence bispectrum and in Takada \& Jain (2003a), Zaldarriaga \& Scoccimarro (2003) for the shear correlation functions. The accuracy of the model has been extensively commented on in those papers and in Fosalba et al. (2005).

The basic relation for computing the correlation functions in the one-halo model has been given by Scoccimarro (1997, taking advantage of a result given by Davydychev 1992) as discussed in Zaldarriaga \& Scoccimarro (2003).

The one-halo amounts to assuming that a stochastic field $\phi$ can be represented by a randomly located halo with a spherically symmetric $\phi$ profile, $\phi(\boldsymbol{r}) \sim F\left(\left|\boldsymbol{r}-\boldsymbol{r}_{0}\right|\right)$ where $\boldsymbol{r}_{0}$, the center of the halo is evenly distributed in space. The $\phi$ correlation functions are then,

$$
\begin{aligned}
F_{n}\left(\boldsymbol{r}_{1} \ldots \boldsymbol{r}_{n}\right)= & \left\langle\phi\left(\boldsymbol{r}_{1}\right) \ldots \phi\left(\boldsymbol{r}_{n}\right)\right\rangle \sim \\
& \int \mathbf{d}^{d} \boldsymbol{r}_{0} F\left(\left|\boldsymbol{r}_{1}-\boldsymbol{r}_{0}\right|\right) \ldots F\left(\left|\boldsymbol{r}_{n}-\boldsymbol{r}_{0}\right|\right) .
\end{aligned}
$$

This expression is in general complicated. We are interested in such an expression for the three-point function and in the case of a power law profile, $F(x) \sim x^{-\alpha}$. Such an integral converges for $d-3 \alpha<0$ and $d-\alpha>0$. For $F(x)=F_{0} x^{-\alpha}$ the final result can be given in a closed form in terms of hypergeometric functions. This result has been presented by Scoccimarro (1997) for the three-point function taking advantage of integrals developed in the context of high energy physics.

We are interested in this result in the context of a $2 \mathrm{D}$ projected density field ${ }^{1}$. The result reads

$$
\begin{aligned}
& F_{3}\left(\boldsymbol{r}_{1}, \boldsymbol{r}_{2}, \boldsymbol{r}_{3}\right)= \\
& \quad \frac{\pi^{3} l_{3}^{2-3 \alpha}}{2 \sin (\alpha \pi / 2)^{2} \cos (\alpha \pi / 2) \Gamma(2-3 \alpha / 2) \Gamma(\alpha / 2)^{2}} \\
& \quad \times\left[\Gamma(\alpha / 2) \Gamma(1-3 \alpha / 2) G\left(z_{3}\right) G\left(\bar{z}_{3}\right)\right. \\
& \left.\quad-\Gamma(2-3 \alpha / 2) \Gamma(1-\alpha / 2) H\left(z_{3}\right) H\left(\bar{z}_{3}\right)\right]
\end{aligned}
$$

where the variables are those defined in the previous part and where $G(z)$ and $H(z)$ are respectively,

$$
\begin{aligned}
& G(z)=(1-z)^{1-\alpha} \frac{{ }_{2} F_{1}\left(\frac{\alpha}{2}, 1-\frac{\alpha}{2}, \alpha, z\right)}{\Gamma(\alpha)} \\
& H(z)=z^{1-\alpha} \frac{{ }_{2} F_{1}\left(\frac{\alpha}{2}, 1-\frac{\alpha}{2}, 2-\alpha, z\right)}{\Gamma(2-\alpha)} .
\end{aligned}
$$

This expression results from a slight modification of the formula presented in Scoccimarro (1997). From the form (15) it is clear that $F_{3}$ is real.

The idea pursued in this paper is that convergence fields can be described to a reasonable accuracy with the one-halo model with a density profile close to $\alpha=1$. The goal though is not to use Eq. (15) for the convergence field but to construct in this framework the shear three-point functions,

$$
\xi_{\gamma_{i} \gamma_{j} \gamma_{k}}\left(\boldsymbol{r}_{1}, \boldsymbol{r}_{2}, \boldsymbol{r}_{3}\right)=\left\langle\gamma_{i}\left(\boldsymbol{r}_{1}\right) \gamma_{j}\left(\boldsymbol{r}_{1}\right) \gamma_{k}\left(\boldsymbol{r}_{1}\right)\right\rangle
$$

\footnotetext{
1 The scales at which the correlations are to be measured are small enough so that the small angle approximation can be used.
}

where $i, j, k$ stand for the two component indices of the shear field.

To obtain this function two routes can be followed. The one advocated in Zaldarriaga \& Scoccimarro (2003) is to transform the formal expression of $\xi_{\gamma_{i} \gamma_{j} \gamma_{k}}$ into a sum of integrals, each of which can be computed in a manner similar to $F_{3}$. It leads however to expressions with a very large number of terms that are hard to manipulate and simplify.

The route followed here is radically different. As suggested in the second section it takes proper subsequent derivatives of the potential three-point function, $\xi_{\phi \phi \phi}\left(\boldsymbol{r}_{1}, \boldsymbol{r}_{2}, \boldsymbol{r}_{3}\right)$ with respect to the positions. The potential correlation function is a priori not well defined because it requires the use of Eq. (15) when $\alpha$ is about -1 . It demands then a regularization of the profile at large distances. It can be checked however that the introduction of this cutoff does not affect the finite distance behavior of its derivatives if those are finite.

The general mathematical operators to be used have been presented in the previous section. The calculations remain tractable in the one-halo model case because the functions $G$ and $H$ have simple derivatives. It can be shown that (using Gradshteyn \& Ryzhik 1980, Eqs. (9.137.6), (9137.7) and (9.137.17))

$$
\begin{aligned}
& \frac{\mathrm{d}^{n}}{\mathrm{~d} z^{n}} G(z)=G^{(n)}(z)=(1-z)^{1-\alpha-n} s \\
& \quad \times \frac{{ }_{2} F_{1}\left(\frac{\alpha}{2}, 1-\frac{\alpha}{2}, \alpha+n, z\right)}{\Gamma(\alpha+n)} \frac{\Gamma(\alpha / 2+n)}{\Gamma(\alpha / 2)} \frac{\Gamma(1-\alpha / 2+n)}{\Gamma(1-\alpha / 2)} \\
& \frac{\mathrm{d}^{n}}{\mathrm{~d} z^{n}} H(z)=H^{(n)}(z)= \\
& z^{1-\alpha-n} \frac{{ }_{2} F_{1}\left(\frac{\alpha}{2}, 1-\frac{\alpha}{2}, 2-\alpha-n, z\right)}{\Gamma(2-\alpha-n)} .
\end{aligned}
$$

To get the shear correlation functions the subsequent steps should be followed,

$$
\begin{aligned}
& \xi_{\phi \phi \gamma}\left(l_{3}, z_{3}\right)=\frac{1}{l_{3}^{2}} \partial_{z_{3}^{2}} \xi_{\phi \phi \phi}\left(l_{3}, z_{3}\right) \\
& \xi_{\phi \gamma \gamma}\left(l_{2}, z_{2}\right)=\frac{1}{l_{2}^{2}} \partial_{z_{2}}^{2}\left\{R_{32} \xi_{\phi \phi \gamma}\left(l_{2}, z_{2}\right)\right\} \\
& \xi_{\gamma \gamma \gamma}\left(l_{1}, z_{1}\right)=R_{13}^{3} \frac{1}{l_{2}^{2}} \partial_{z_{1}}^{2}\left\{R_{21}^{2} \xi_{\phi \gamma \gamma}\left(l_{1}, z_{1}\right)\right\}
\end{aligned}
$$

and a similar expression for the other components of the shear correlation functions, $\xi_{\gamma\left(\boldsymbol{r}_{1}\right) \gamma\left(\boldsymbol{r}_{2}\right) \bar{\gamma}\left(\boldsymbol{r}_{3}\right)}, \boldsymbol{\xi}_{\gamma\left(\boldsymbol{r}_{1}\right) \bar{\gamma}\left(\boldsymbol{r}_{2}\right) \bar{\gamma}\left(\boldsymbol{r}_{3}\right)}$, etc. Note that in those expressions a change of variable should be applied from one line to another (for both the $z$ and the $l$ variables); the rotation operators are $R_{21}=1 / R_{12}=\left(1-\bar{z}_{1}\right) /\left(1-z_{1}\right)$, $R_{13}=1 / R_{31}=\left(1-\bar{z}_{3}\right) /\left(1-z_{3}\right), R_{32}=R_{31} R_{12}=\left(1-z_{3}\right) /(1-$ $\left.\bar{z}_{3}\right)\left(1-z_{1}\right) /\left(1-\bar{z}_{1}\right)$ and they should be replaced by their inverse (or equivalently their complex conjugate) each time one conjugates components of the shear.

After calculations and simplification taking advantage of recursion relations of the hypergeometric functions and with 
Table 1. Expression of four components of the shear three-point function where $C_{\alpha}\left(l_{3}\right)=-l_{3}^{-4-3 \alpha} \pi^{3} \csc \left(\frac{\alpha \pi}{2}\right)^{2} \sec \left(\frac{\alpha \pi}{2}\right) /\left(2 \Gamma\left(2-\frac{3 \alpha}{2}\right) \Gamma\left(\frac{\alpha}{2}\right)^{3}\right)$.

$$
\begin{aligned}
& \xi_{\gamma\left(\boldsymbol{r}_{1}\right) \gamma\left(\boldsymbol{r}_{2}\right) \gamma\left(\boldsymbol{r}_{3}\right)}=-\frac{C_{\alpha}\left(l_{3}\right) \alpha^{2}(2+\alpha)^{2}}{\left(-1+z_{3}\right)^{3} z_{3}^{3}}\left\{\left[2 \alpha\left(1-2 z_{3}\right)^{2}\left(-2-z_{3}+z_{3}^{2}\right)+8\left(1-2 z_{3}+4 z_{3}^{3}-2 z_{3}^{4}\right)\right.\right. \\
& \left.+3 \alpha^{2}\left(-4+5 z_{3}+3 z_{3}^{2}-16 z_{3}^{3}+8 z_{3}^{4}\right)\right] \Gamma\left(\frac{\alpha}{2}\right) \Gamma\left(-1+\frac{3 \alpha}{2}\right) G^{(0)}\left(z_{3}\right) G^{(0)}\left(\bar{z}_{3}\right) \\
& +8(1+\alpha)\left(2-5 z_{3}+2 z_{3}^{2}+2 z_{3}^{3}-5 z_{3}^{4}+2 z_{3}^{5}\right) \Gamma\left(\frac{\alpha}{2}\right) \Gamma\left(-1+\frac{3 \alpha}{2}\right) G^{(1)}\left(z_{3}\right) G^{(0)}\left(\bar{z}_{3}\right) \\
& -\Gamma\left(2-\frac{3 \alpha}{2}\right) \Gamma\left(1-\frac{\alpha}{2}\right)\left[\left(2 \alpha\left(1-2 z_{3}\right)^{2}\left(-2-z_{3}+z_{3}^{2}\right)+8\left(1-2 z_{3}+4 z_{3}^{3}-2 z_{3}^{4}\right)\right.\right. \\
& \left.+3 \alpha^{2}\left(-4+5 z_{3}+3 z_{3}^{2}-16 z_{3}^{3}+8 z_{3}^{4}\right)\right) H^{(0)}\left(z_{3}\right) \\
& \left.\left.+8(1+\alpha)\left(2-5 z_{3}+2 z_{3}^{2}+2 z_{3}^{3}-5 z_{3}^{4}+2 z_{3}^{5}\right) H^{(1)}\left(z_{3}\right)\right] H^{(0)}\left(\bar{z}_{3}\right)\right\} \\
& \xi_{\gamma\left(\boldsymbol{r}_{1}\right) \gamma\left(\boldsymbol{r}_{2}\right) \bar{\gamma}\left(\boldsymbol{r}_{3}\right)}=-\frac{C_{\alpha}\left(l_{3}\right) \alpha^{2}(2+\alpha)}{\left(-1+z_{3}\right) z_{3}\left(-1+\bar{z}_{3}\right) \bar{z}_{3}}\left\{-\left[\Gamma ( \frac { \alpha } { 2 } ) \Gamma ( - 1 + \frac { 3 \alpha } { 2 } ) \left(( - 2 + 3 \alpha ) \left(8\left(-1+z_{3}\right) z_{3}\right.\right.\right.\right. \\
& \left.+\alpha\left(-1-8 z_{3}+8 z_{3}^{2}\right)\right) G^{(0)}\left(z_{3}\right)+4\left(-1+2 z_{3}\right)\left(2\left(-1+z_{3}\right) z_{3}\right. \\
& \left.\left.\left.+\alpha\left(-1-2 z_{3}+2 z_{3}^{2}\right)\right) G^{(1)}\left(z_{3}\right)\right)\left((-2+3 \alpha) G^{(0)}\left(\bar{z}_{3}\right)+4\left(-1+2 \bar{z}_{3}\right) G^{(1)}\left(\bar{z}_{3}\right)\right)\right] \\
& +\Gamma\left(2-\frac{3 \alpha}{2}\right) \Gamma\left(1-\frac{\alpha}{2}\right)\left[(-2+3 \alpha)\left(8\left(-1+z_{3}\right) z_{3}+\alpha\left(-1-8 z_{3}+8 z_{3}^{2}\right)\right) H^{(0)}\left(z_{3}\right)\right. \\
& \left.+4\left(-1+2 z_{3}\right)\left(2\left(-1+z_{3}\right) z_{3}+\alpha\left(-1-2 z_{3}+2 z_{3}^{2}\right)\right) H^{(1)}\left(z_{3}\right)\right]\left((-2+3 \alpha) H^{(0)}\left(\bar{z}_{3}\right)\right. \\
& \left.\left.+4\left(-1+2 \bar{z}_{3}\right) H^{(1)}\left(\bar{z}_{3}\right)\right)\right\} \\
& \xi_{\gamma\left(r_{1}\right) \bar{\gamma}\left(r_{2}\right) \gamma\left(r_{3}\right)}=\frac{C_{\alpha}\left(l_{3}\right) \alpha(2+\alpha)}{\left(-1+z_{3}\right)^{3} z_{3} \bar{z}_{3}}\left\{-2 \Gamma\left(1+\frac{\alpha}{2}\right) \Gamma\left(-1+\frac{3 \alpha}{2}\right)\left[(-2+3 \alpha)\left(-4 z_{3}+\alpha\left(-1-5 z_{3}+2 z_{3}^{2}\right)\right)\right.\right. \\
& \left.\times G^{(0)}\left(z_{3}\right)+4\left(1+z_{3}\right)\left(-2 z_{3}+\alpha\left(1-4 z_{3}+z_{3}^{2}\right)\right) G^{(1)}\left(z_{3}\right)\right] \\
& \times\left[(-2+3 \alpha)\left(1+2 \bar{z}_{3}\right) G^{(0)}\left(\bar{z}_{3}\right)+4\left(-1+\bar{z}_{3}^{2}\right) G^{(1)}\left(\bar{z}_{3}\right)\right] \\
& +\alpha \Gamma\left(2-\frac{3 \alpha}{2}\right) \Gamma\left(1-\frac{\alpha}{2}\right)\left[(-2+3 \alpha)\left(-4 z_{3}+\alpha\left(-1-5 z_{3}+2 z_{3}^{2}\right)\right) H^{(0)}\left(z_{3}\right)\right. \\
& \left.+4\left(1+z_{3}\right)\left(-2 z_{3}+\alpha\left(1-4 z_{3}+z_{3}^{2}\right)\right) H^{(1)}\left(z_{3}\right)\right] \\
& \left.\times\left[(-2+3 \alpha)\left(1+2 \bar{z}_{3}\right) H^{(0)}\left(\bar{z}_{3}\right)+4\left(-1+\bar{z}_{3}^{2}\right) H^{(1)}\left(\bar{z}_{3}\right)\right]\right\} \\
& \xi_{\gamma\left(r_{1}\right) \bar{\gamma}\left(r_{2}\right) \bar{\gamma}\left(r_{3}\right)}=\frac{C_{\alpha}\left(l_{3}\right) \alpha(2+\alpha)}{\left(-1+z_{3}\right)\left(-1+\bar{z}_{3}\right) \bar{z}_{3}^{3}}\left\{2 \Gamma ( 1 + \frac { \alpha } { 2 } ) \Gamma ( - 1 + \frac { 3 \alpha } { 2 } ) \left[\left(-6+\alpha\left(9-6 z_{3}\right)+4 z_{3}\right) G^{(0)}\left(z_{3}\right)\right.\right. \\
& \left.-4\left(-2+z_{3}\right) z_{3} G^{(1)}\left(z_{3}\right)\right]\left[(-2+3 \alpha)\left(4\left(-1+\bar{z}_{3}\right)+\alpha\left(-4+\bar{z}_{3}+2 \bar{z}_{3}^{2}\right)\right) G^{(0)}\left(\bar{z}_{3}\right)\right. \\
& \left.+4\left(-2+\bar{z}_{3}\right)\left(2\left(-1+\bar{z}_{3}\right)+\alpha\left(-2+2 \bar{z}_{3}+\bar{z}_{3}^{2}\right)\right) G^{(1)}\left(\bar{z}_{3}\right)\right]+\alpha \Gamma\left(2-\frac{3 \alpha}{2}\right) \Gamma\left(1-\frac{\alpha}{2}\right) \\
& \times\left[(-2+3 \alpha)\left(-3+2 z_{3}\right) H^{(0)}\left(z_{3}\right)+4\left(-2+z_{3}\right) z_{3} H^{(1)}\left(z_{3}\right)\right] \\
& \times\left[(-2+3 \alpha)\left(4\left(-1+\bar{z}_{3}\right)+\alpha\left(-4+\bar{z}_{3}+2 \bar{z}_{3}^{2}\right)\right) H^{(0)}\left(\bar{z}_{3}\right)\right. \\
& \left.\left.+4\left(-2+\bar{z}_{3}\right)\left(2\left(-1+\bar{z}_{3}\right)+\alpha\left(-2+2 \bar{z}_{3}+\bar{z}_{3}^{2}\right)\right) H^{(1)}\left(\bar{z}_{3}\right)\right]\right\}
\end{aligned}
$$

the help of formal calculators one can obtain explicit expressions for the shear correlation functions. The first four are given in Table 1 and the other four are obtained as complex conjugates.

The results are given as a function of $z_{3}$ and $\bar{z}_{3}$ and therefore when the coordinates (in particular the shear coordinates) are expressed in the $\left(\boldsymbol{r}_{1}, \boldsymbol{r}_{2}\right)$ basis.

\section{Shear patterns in the one-halo model}

The amplitude of the shear correlation function can then be compared to the amplitude of the convergence correlation function. Consider $\xi_{\gamma}(z)$ defined as

$\xi_{\gamma}(z)=\sqrt{\sum_{i j k} \xi_{\gamma_{i}}\left(\boldsymbol{r}_{1}\right) \gamma_{j}\left(\boldsymbol{r}_{2}\right) \gamma_{k}\left(\boldsymbol{r}_{3}\right) \xi_{\gamma_{i}}\left(\boldsymbol{r}_{1}\right) \gamma_{j}\left(\boldsymbol{r}_{2}\right) \gamma_{k}\left(\boldsymbol{r}_{3}\right)}$. 

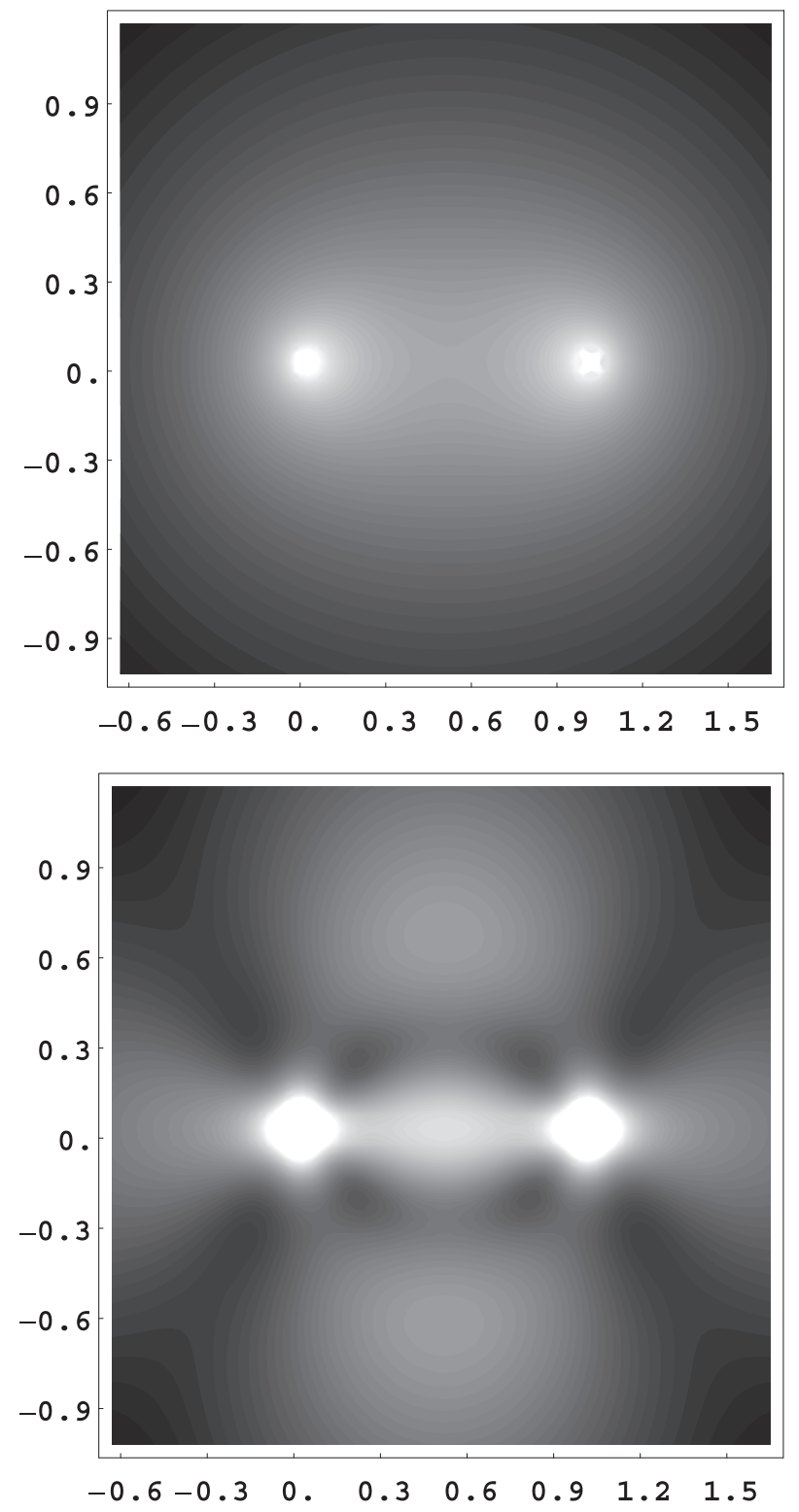

Fig. 1. Comparison of the spatial dependence of the amplitude of the three-point function for both the convergence field (top panel) and the shear field (bottom panel). These results correspond to the one-halo model with $\alpha=-1$. In this plot, $\boldsymbol{r}_{1}=(0,0)$ and $\boldsymbol{r}_{2}=(1,0)$. As can be observed the spatial structure of the shear three-point function is much more complex than the one of the convergence field.

There are some symmetry properties in this quantity due to the fact that it should be invariant under $z \rightarrow 1 /(1-z)$ and $z \rightarrow 1-1 / z$ when properly rescaled (these changes correspond to a transform that leaves the triangle shape unchanged; the amplitude of the shear functions are then only changed by a scale factor effect).

Its amplitude in shown in Fig. 1 where the spatial dependence of the amplitude of the shear correlation function is compared to that of the convergence correlation function. While the convergence correlation function has a very simple spatial dependence, the spatial dependence of the shear correlation is much more complex, exhibiting minima for certain configurations. These patterns are quite dependent on the power
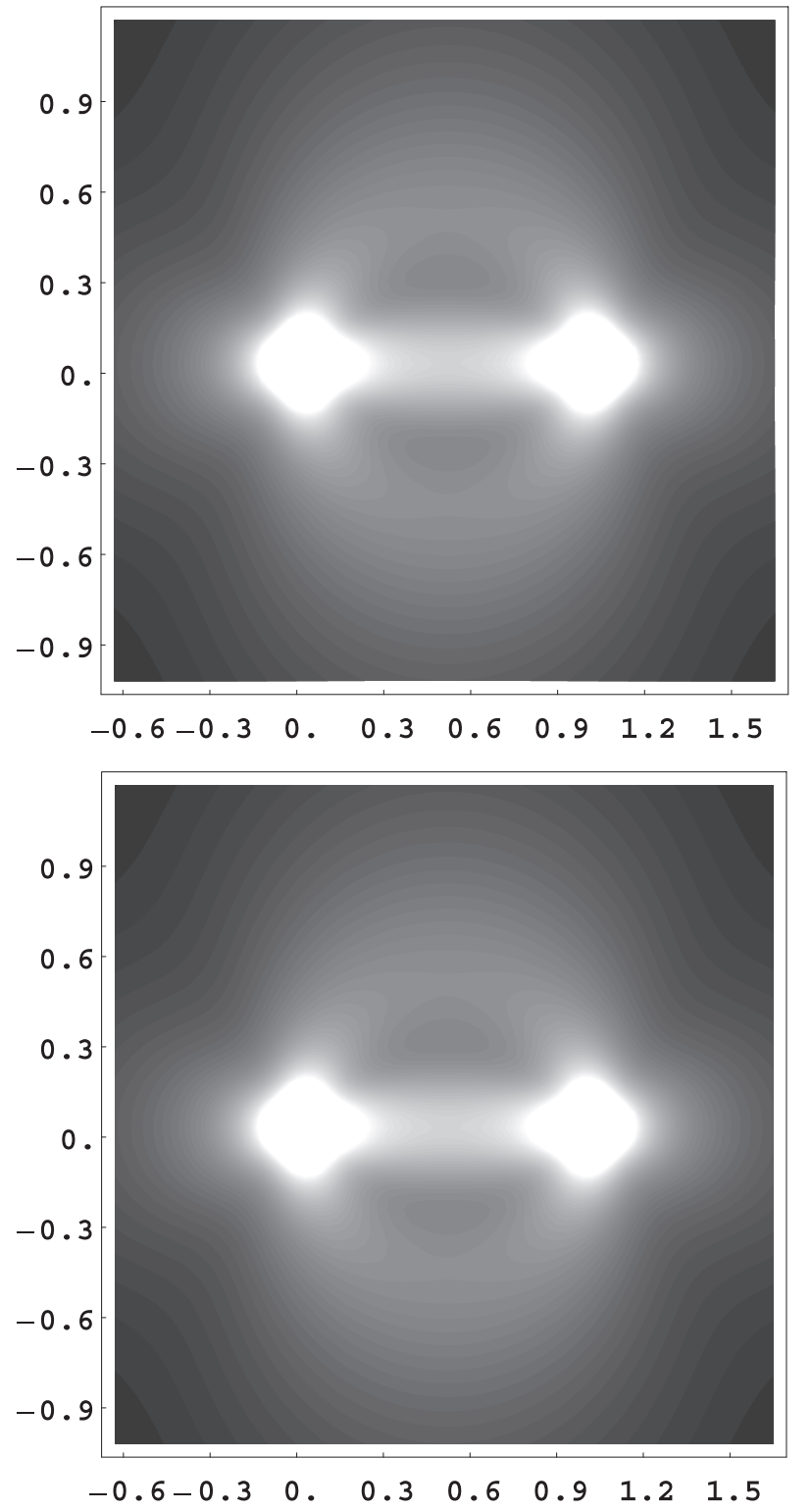

Fig. 2. Comparison of the spatial dependence of the amplitude of the three-point function for $\alpha=-0.75$ (top panel) and $\alpha=-0.5$ (bottom panel).

spectrum index (right panel of Figs. 1 and 2). In particular, the existence of a local maximum of the signal for equilateral triangles vanishes for steep spectra.

A fair fraction of the signal however can be extracted from a subpart of the correlation functions. Following Bernardeau et al. (2003) it is possible to map at least some aspects of the shear patterns while considering the function,

$\xi_{3}\left(\boldsymbol{r}_{3}\right)=\left\langle\left(\gamma_{\boldsymbol{r}_{1}} \cdot \gamma_{\boldsymbol{r}_{2}}\right) \gamma_{\boldsymbol{r}_{3}}\right\rangle$

which can be written in terms of the complex coordinate correlations as

$\xi_{3}\left(\boldsymbol{r}_{3}\right)=\frac{1}{2}\left(\xi_{\gamma\left(\boldsymbol{r}_{1}\right) \bar{\gamma}\left(\boldsymbol{r}_{2}\right) \gamma\left(\boldsymbol{r}_{3}\right)}+\xi_{\bar{\gamma}\left(\boldsymbol{r}_{1}\right) \gamma\left(\boldsymbol{r}_{2}\right) \gamma\left(\boldsymbol{r}_{3}\right)}\right)$.

The $\xi_{3}$ patterns are depicted in Fig. 3. They are very similar to what has been observed in numerical simulations. This 

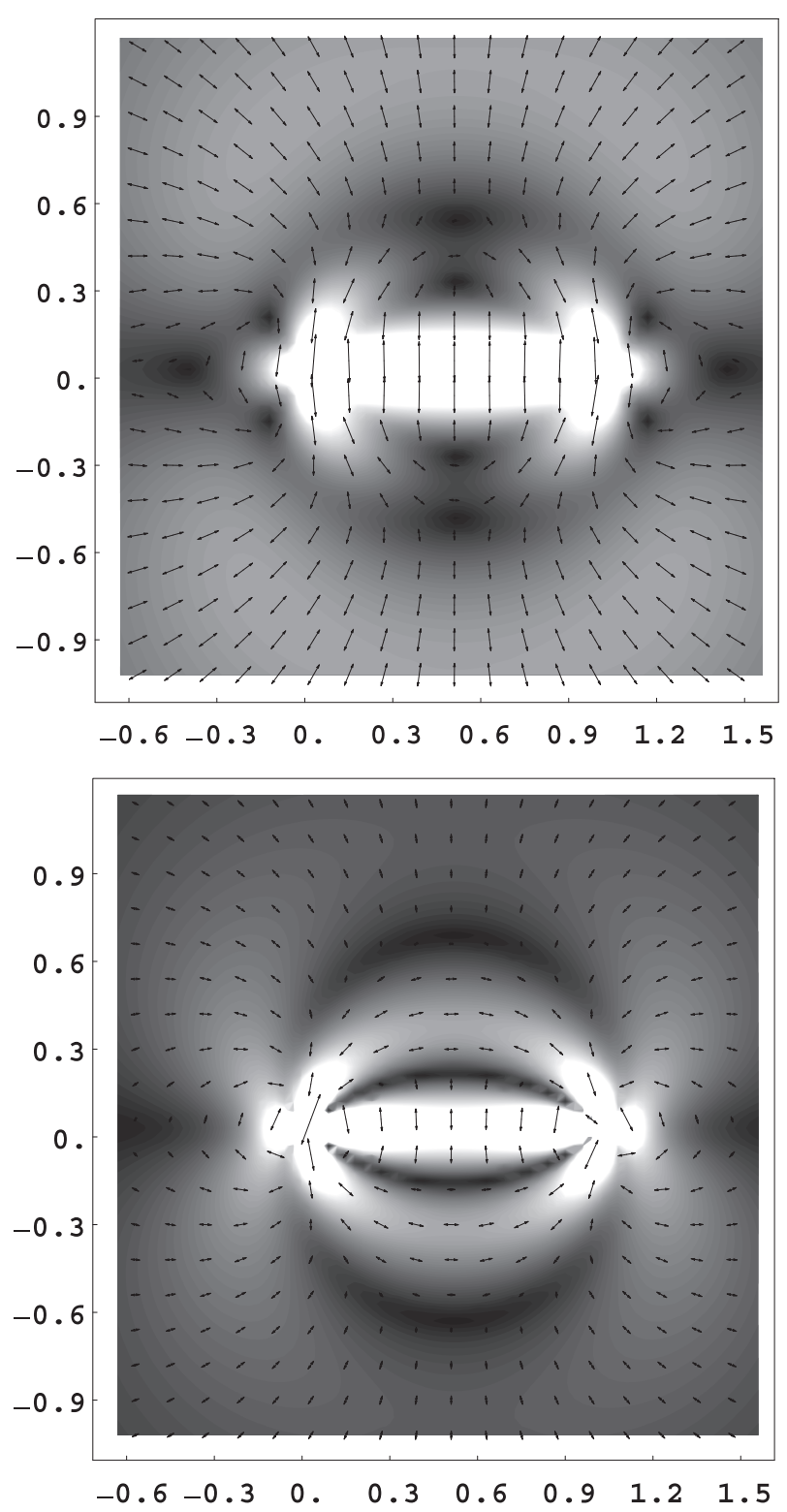

Fig. 3. Pattern of the $\xi_{3}$ field in the case of the one-halo model for $l_{3}=1, \alpha=-1$ (top panel) and $\alpha=-0.5$ (bottom panel). $\boldsymbol{r}_{1}=(0,0)$ and $\boldsymbol{r}_{2}=(1,0)$. The level indicates the amplitude of this component of the shear correlation.

part of the components however does not encompass the signal coming from equilateral configurations. The extension of the uniform and strong pattern in between the two points depends again on the halo profile.

\section{Prospects}

The use of complex coordinates proved fruitful in computations of shear correlation functions. It has been possible to obtain the shear three-point correlation functions in manageable forms for the one-halo model with a power law profile. In the context of this model those results can be used to infer general properties for the shear correlation patterns, as in Benabed \& Scoccimarro (2005); this is a first step toward the construction of robust methods for the measurement of the shear three-point function.

The theoretical framework presented in this paper is very general and can be used for alternative descriptions of the density correlation properties such as those used in the quasilinear or the nonlinear regime. Indeed the mathematical relation between the projected potential correlation functions and those of the projected density is rather simple and could be integrated out for simple cases ${ }^{2}$ opening the way for explicit computations of the shear correlation functions in more diverse cases.

Acknowledgements. The calculations presented in this paper would not have been possible without the formula initially re-discovered by Román Scoccimarro.

\section{References}

Benabed, K., \& Scoccimarro, R. 2005, [arXiv:astro-ph/0505284] Bernardeau, F., Van Waerbeke, L., \& Mellier, Y. 1997, A\&A, 322, 1

Bernardeau, F., Colombi, S., Gaztañaga, E., \& Scoccimarro, R. 2002a, Phys. Rep., 367, 1

Bernardeau, F., Mellier, Y., \& van Waerbeke, L. 2002b, A\&A, 389, L28

Bernardeau, F., van Waerbeke, L., \& Mellier, Y. 2003, A\&A, 397, 405

Bernardeau, F. 2004, [arXiv: astro-ph/0409224]

Cooray, A., \& Hu, W. 2001, ApJ, 548, 7

Cooray, A., \& Sheth, R. 2002, Phys. Rep., 372, 1

Davydychev, A. I. 1992, J. Phys. A, 25, 5587

Dolney, D., Jain, B., \& Takada, M. 2004, MNRAS, 352, 1019

Fosalba, P., Pan, J., \& Szapudi, I. 2005 [arXiv: astro-ph/0504305]

Gradshteyn, I. S., \& Ryzhik, J. M. 1980, Table of Integrals, Series and Product (New-York: Academic)

Jain, B., \& Seljak, U. 1997, ApJ, 484, 560

Jarvis, M., Bernstein, G., \& Jain, B. 2004, MNRAS, 352, 338

Ma, C.-P., \& Fry, J. N. 2000, ApJ, 543, 503

Mellier, Y. 1999, ARA\&A, 37, 127

Peacock, J. A., \& Smith, R. E. 2000, MNRAS, 318, 1144

Pen, U.-L., Zhang, T., van Waerbeke, L., et al. 2003, ApJ, 592, 664

Scoccimarro, R. 1997, ApJ, 487, 1

Scoccimarro, R., Sheth, R. K., Hui, L., \& Jain, B. 2001, ApJ, 546, 20

Seljak, U. 2000, MNRAS, 318, 203

Schneider, P., \& Lombardi, M. 2003, A\&A, 397, 809

Sheth, R. K., \& Jain, B. 1997, MNRAS, 285, 231

Takada, M., \& Jain, B. 2003a, ApJ, 583, L49

Takada, M., \& Jain, B. 2003b, MNRAS, 344, 857

Zaldarriaga, M., \& Scoccimarro, R. 2003, ApJ, 584, 559

\footnotetext{
${ }^{2}$ It is to be noted however that the potential correlations are not uniquely defined from those of the projected density field.
} 